\title{
THE FORMATION OF BINARY STARS
}

IAU SYMPOSIUM VOLUME 200

\section{COVER ILLUSTRATION:}
A binary system with mass ratio $M_{2} / M_{1}=0.75$ as it accretes gas from an infalling envelope. The angular momentum of the gas is sufficient for some of it to settle into a circumbinary disc. The circumstellar discs are fed both directly from the infalling envelope and via streams from the circumbinary disc. The image is very similar to the pre-main-sequence binary system, GG Tauri.
Reference: Bate M.R. 2000, MNRAS, 314, 33 (Figure 8) 


\title{
THE ASTRONOMICAL SOCIETY OF THE PACIFIC \\ 390 Ashton Avenue - San Francisco, California - USA 94112-1722 \\ Phone: (415) 337-1100 \\ Fax: (415) 337-5205 \\ E-Mail: catalog@aspsky.org \\ Web Site: www.aspsky.org
}

\section{Publisher}

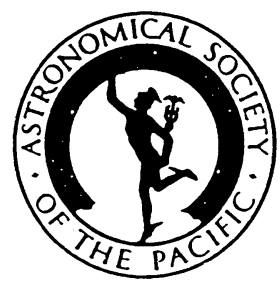

\section{ASP CONFERENCE SERIES - EDITORIAL STAFF}

Managing Editor: D. H. McNamara

Associate Managing Editor: J. W. Moody
LaTeX-Computer Consultant: T. J. Mahoney

Production Manager: Enid L. Livingston

PO Box 24453, 211 KMB, Brigham Young University, Provo, Utah, 84602-4463

Phone: (801) 378-2111 Fax: (801) 378-4049 E-Mail: pasp@byu.edu

\author{
ASP CONFERENCE SERIES PUBLICATION COMMITTEE: \\ Alexei V. Filippenko Geoffrey Marcy \\ Ray Norris \\ Donald Terndrup \\ Frank X. Timmes \\ C. Megan Urry
}

A listing of all other ASP Conference Series Volumes and IAU Volumes published by the ASP is cited at the back of this volume 


\section{INTERNATIONAL ASTRONOMICAL UNION \\ 98bis, Bd Arago - F-75014 Paris - France \\ Tel: +33 $143258358 \quad$ E-mail: iau@iap.fr \\ Fax: +33 143252616 Web Site: www.iau.org}

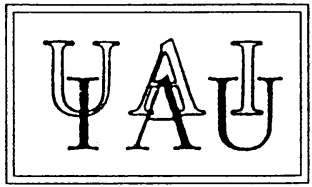

\section{THE FORMATION OF BINARY STARS}

Proceedings of the $200^{\mathrm{TH}}$ Symposium of the

International Astronomical Union

held in Potsdam, Germany

10 -15 April 2000

Edited by

HANS ZINNECKER

Astrophysikalisches Institut, Potsdam, Germany

and

ROBERT D. MATHIEU

Department of Astronomy, University of Wisconsin, Wisconsin, USA 
(C) 2001 by International Astronomical Union All Rights Reserved

No part of the material protected by this copyright notice may be reproduced or utilized in any form or by any means - graphic, electronic, or mechanical including photocopying, taping, recording or by any information storage and retrieval system, without written permission from the IAU:

Library of Congress Cataloging in Publication Data

Main entry under title

Card Number: 2001090270

ISBN: $1-58381-068-4$

IAU Publications - First Edition

Published on behalf of IAU by Astronomical Society of the Pacific

Printed in United States of America by Sheridan Books, Chelsea, Michigan 


\section{Table of Contents}

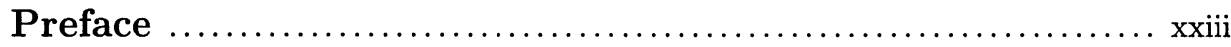

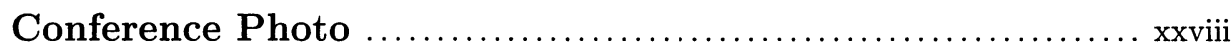

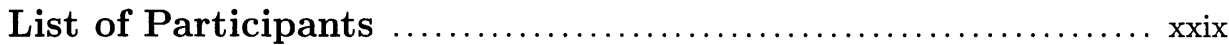

\section{INTRODUCTION}

\section{Binary Stars: Historical Milestones}

\section{H. Zinnecker}

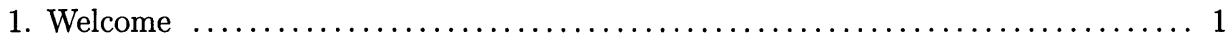

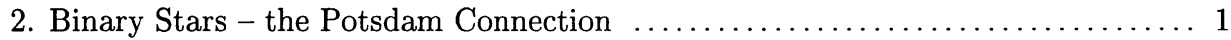

3. The Discovery of Binary Stars - a Retrospective .................... 4

3.1. Classical Studies ......................................... 4

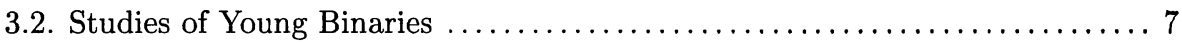

4. Binary Star Formation - a Brief History of Ideas $\ldots \ldots \ldots \ldots \ldots \ldots \ldots \ldots \ldots$

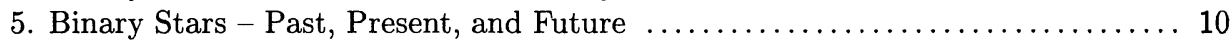

\section{THEORETICAL CONTEXT: THE BIG PICTURE}

\section{Formation of Wide Binaries by Fragmentation}

P. Bodenheimer, A. Burkert

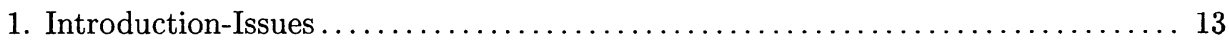

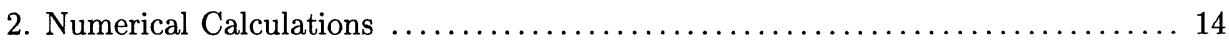

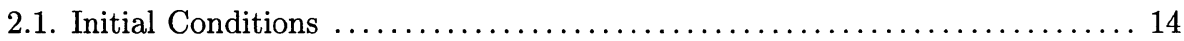

2.2. Is there a Criterion for Fragmentation? ........................ 15

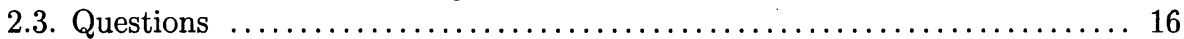

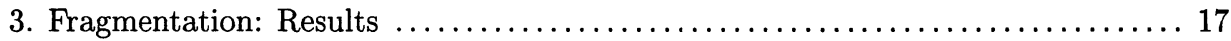

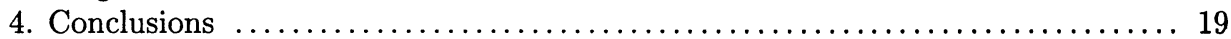

\section{The Formation of Close Binary Stars}

\section{A. Bonnell}

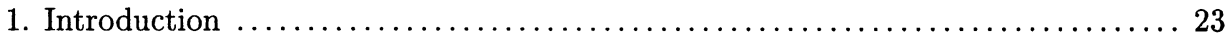

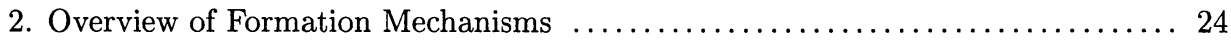

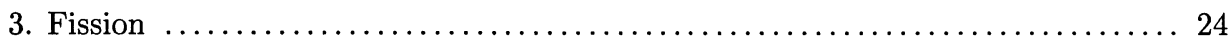

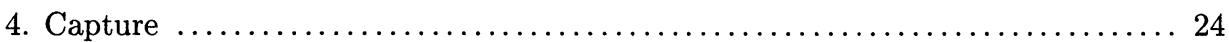

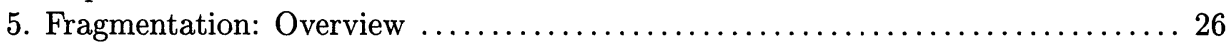

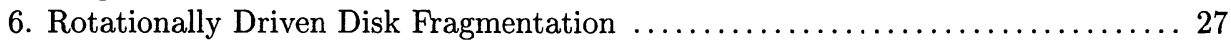

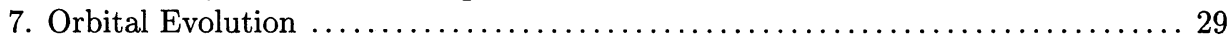

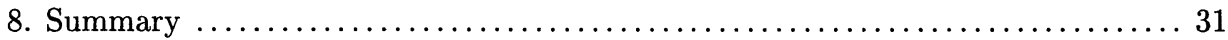




\section{Impulsively Triggered Binary Star Formation}

\section{A. P. Whitworth}

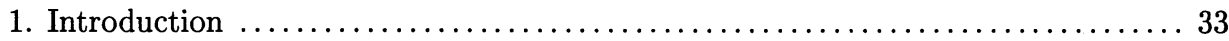

2. Cloud/Cloud Collisions and the Formation of Multiple Protostellar Discs ..... 34

3. Impulsive Interactions between Protostellar Discs $\ldots \ldots \ldots \ldots \ldots \ldots \ldots \ldots \ldots \ldots$

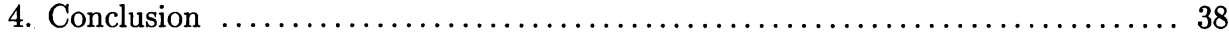

\section{An Update on Binary Formation by Rotational Fission}

\section{J. E. Tohline, R. H. Durisen}

1. Relevant Results up through the 1980 s ........................... 40

2. Results from the Past Decade ............................... 41

2.1. Confirmation of Earlier Dynamic Barlike Mode Results ............. 41

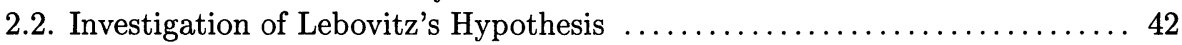

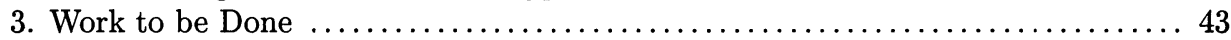

\section{MAIN SEQUENCE BINARY STATISTICS}

\section{Binaries at the Bottom of the Main Sequence and below}

M. Mayor, S. Udry, J.-L. Halbwachs, F. Arenou

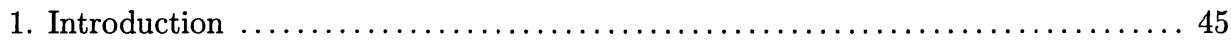

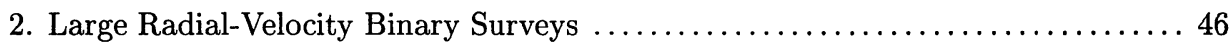

2.1. The CORAVEL G- and K-Dwarf Surveys $\ldots \ldots \ldots \ldots \ldots \ldots \ldots \ldots \ldots \ldots$

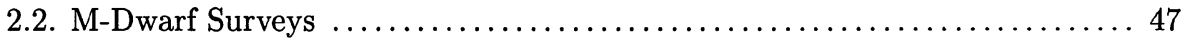

3. Orbital Elements Distributions of Companions to Solar-Type Stars ......... 48

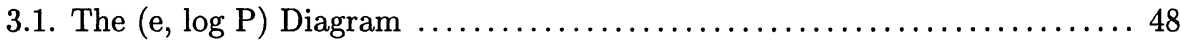

3.2. The Mass-Ratio Distribution: $f(q) \ldots \ldots \ldots \ldots \ldots \ldots \ldots \ldots \ldots \ldots \ldots$

3.3. The Period Distribution and Binary Frequency $\ldots \ldots \ldots \ldots \ldots \ldots \ldots \ldots \ldots$

4. The Mass Function below the Main Sequence $\ldots \ldots \ldots \ldots \ldots \ldots \ldots \ldots \ldots \ldots \ldots$

5. The Mass-Luminosity Relation at the Bottom of the Main Sequence ........ 52

\section{Ultra-Cool Very Low-Mass Binaries}

E. L. Martin, G. Basri

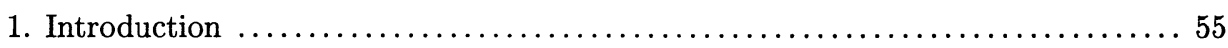

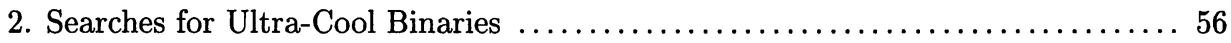

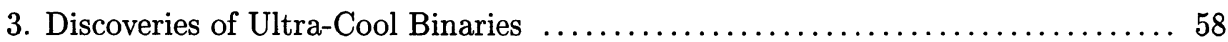

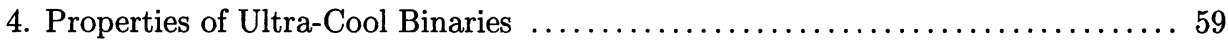

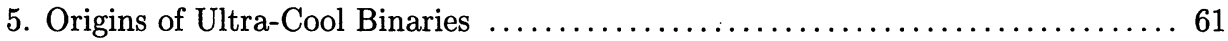

\section{Binarity of Hipparcos Main Sequence Survey Stars}

C. F. Quist, L. Lindegren

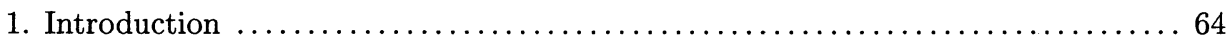

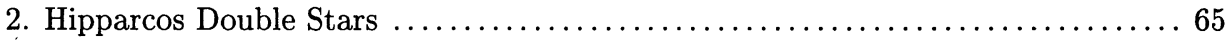




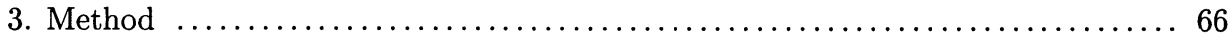

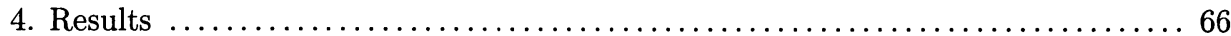

\section{Multiplicity of Massive Stars}

T. Preibisch, G. Weigelt, H. Zinnecker

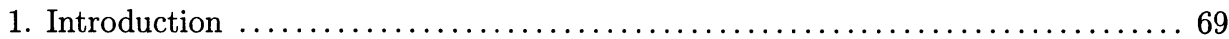

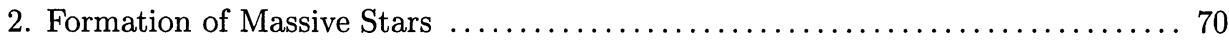

3. Observational Results on OB Star Multiplicity $\ldots \ldots \ldots \ldots \ldots \ldots \ldots \ldots \ldots \ldots \ldots$

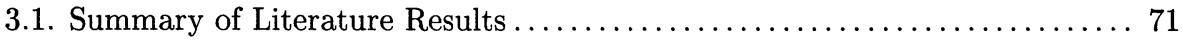

3.2. General Conclusions on OB Star Multiplicity $\ldots \ldots \ldots \ldots \ldots \ldots \ldots \ldots \ldots \ldots$

4. Multiplicity of Massive Stars in the Orion Nebula Cluster $\ldots \ldots \ldots \ldots \ldots \ldots \ldots . \ldots 2$

4.1. Known Companions to the 13 Orion OB Stars...................... 73

4.2. Estimation of the True Number of Companions................... 73

4.3. Properties of the Multiple Systems ........................ 74

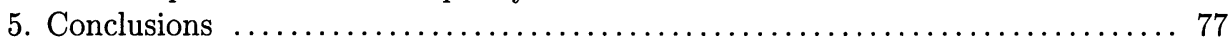

\section{Wolf-Rayet Binaries}

K. A. van der Hucht, B. Hidayat

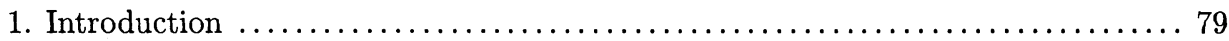

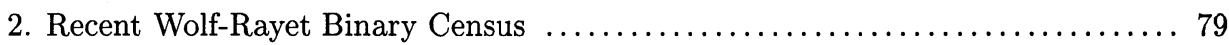

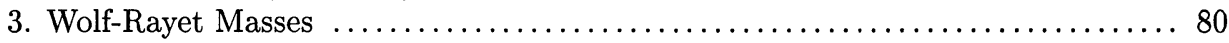

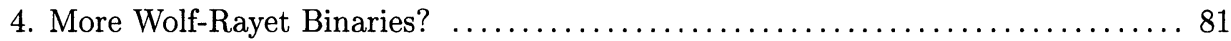

\section{Statistics of Multiple Stars:}

Some Clues to Formation Mechanisms

\section{A. Tokovinin}

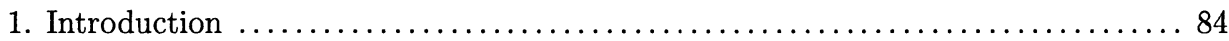

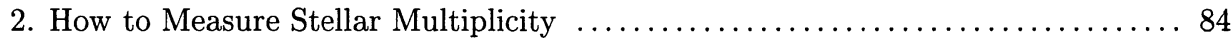

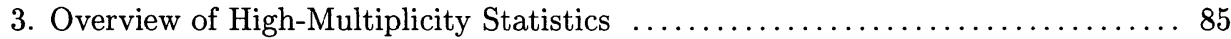

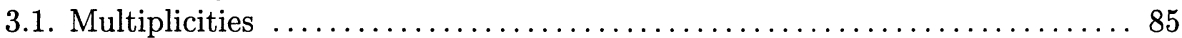

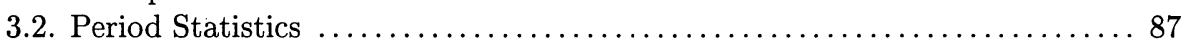

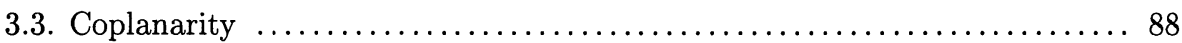

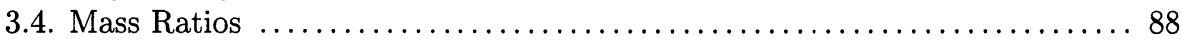

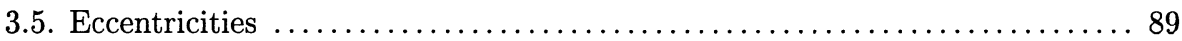

4. Relation of Statistics to Formation/Evolution $\ldots \ldots \ldots \ldots \ldots \ldots \ldots \ldots \ldots \ldots$

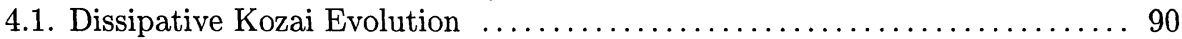

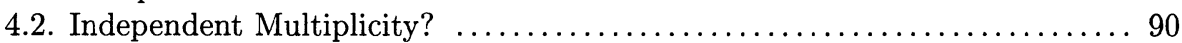

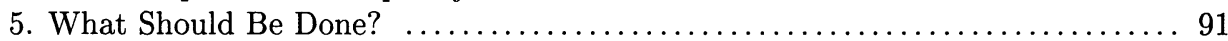

\section{Summary Talk: Implications of Binary Properties for Theories of Star Formation}

\section{R. B. Larson}

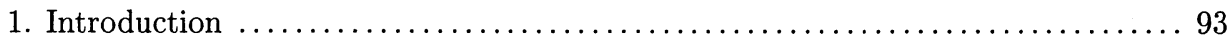

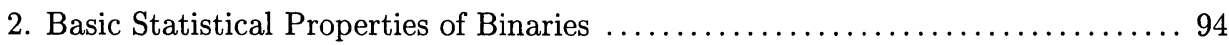

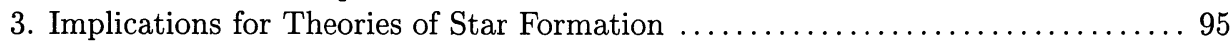




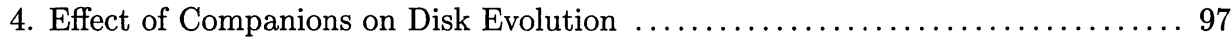

5. Understanding the Broad Period Distribution $\ldots \ldots \ldots \ldots \ldots \ldots \ldots \ldots \ldots \ldots$

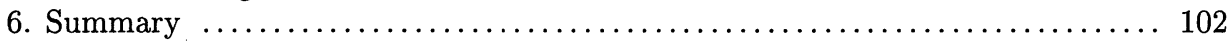

\section{INITIAL CONDITIONS FOR BINARY FORMATION AND PROTOBINARY SYSTEMS}

\section{The Structure and Evolution of Dense Cores}

\section{G. A. Fuller}

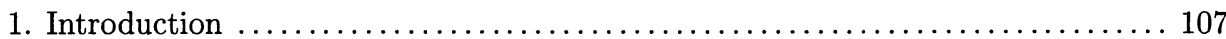

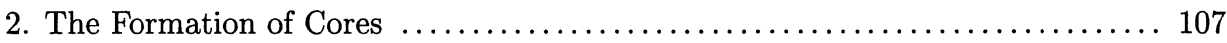

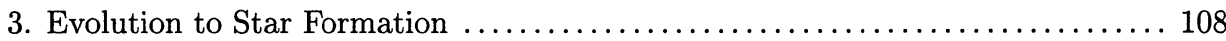

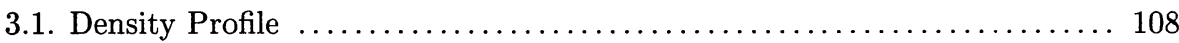

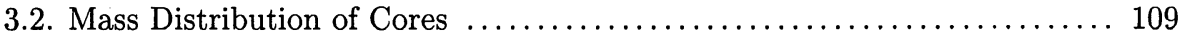

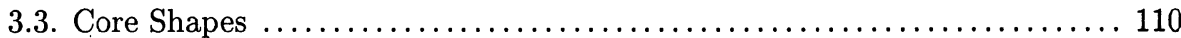

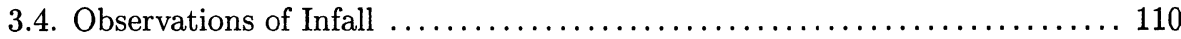

3.5. Angular Momentum ....................................... 112

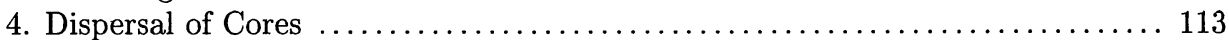

5. Conclusions and Implications for Binary Formation $\ldots \ldots \ldots \ldots \ldots \ldots \ldots \ldots \ldots$

\section{Featured Object: Fragmentation of a Protostellar Core} - the Case of CB 230

R. Launhardt

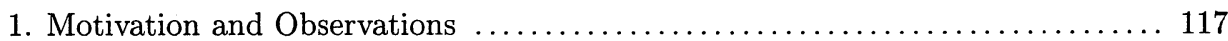

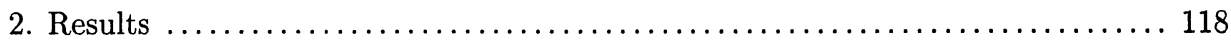

2.1. Morphology and Kinematics of the Double Core .................. 118

2.2. Dust Continuum Emission from Embedded Disk(s) ................ 119

2.3. The Outflow is Also Double .............................. 119

3. CB 230 in the Framework of Binary Star Formation ................. 120

\section{Turbulence and Cloud Angular Momentum}

A. Burkert, P. Bodenheimer

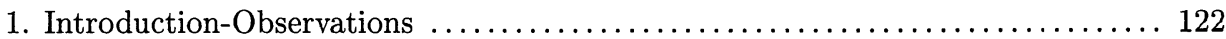

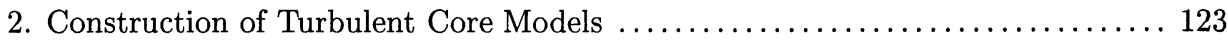

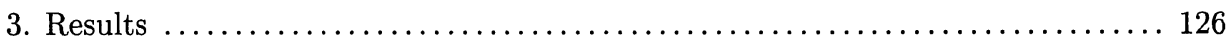

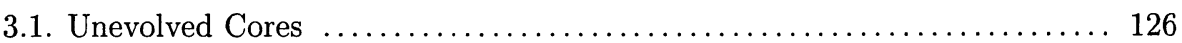

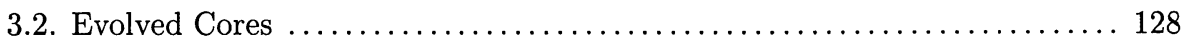

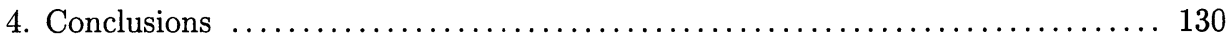

\section{Featured Object: Twin, Aligned Protoplanetary Disks around} the Components of the Young Binary System L1551 IRS5

J. M. Torrelles, L. F. Rodriguez, G. Anglada

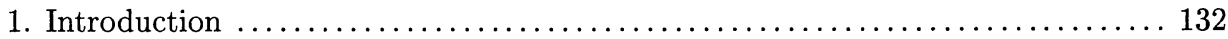

2. What Has Actually Been Observed in L1551 ....................... 133 


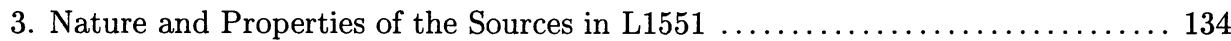

4. General Comments ............................................. 134

\section{Observations of Protobinary Systems}

L. Mundy, L. W. Looney, W. J. Welch

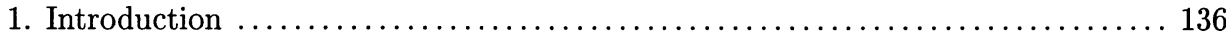

2. The Morphology of Embedded Low Mass Systems $\ldots \ldots \ldots \ldots \ldots \ldots \ldots \ldots \ldots \ldots \ldots$

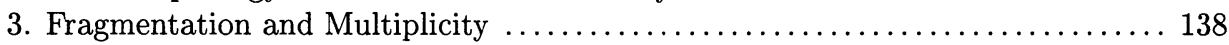

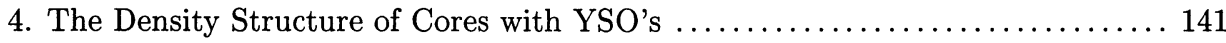

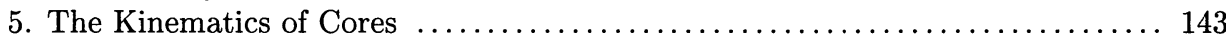

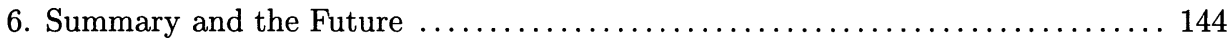

\section{PRE-MAIN SEQUENCE BINARY POPULATIONS}

\section{Multiplicity in T and OB Associations}

R. Köhler, W. Brandner

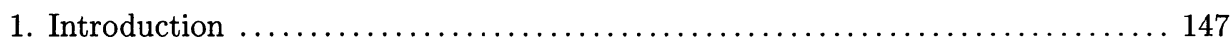

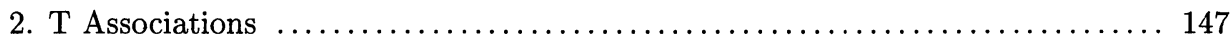

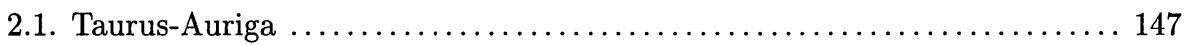

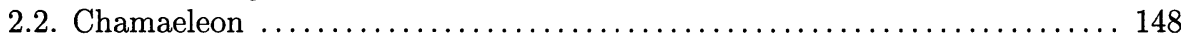

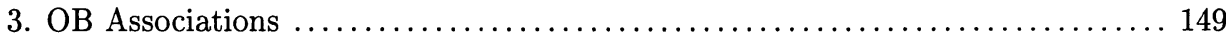

3.1. OB Associations: Why and Where? ............................... 149

3.2. Scorpius-Centaurus: How Do Binaries Form (and Evolve)? ......... 150

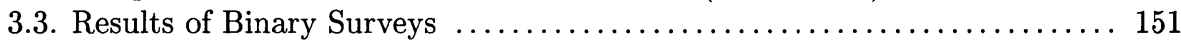

3.4. Binary Properties: A Fossil Record of the Initial Physical Conditions in a Molecular Cloud? ........................................ 152

4. Summary: What Have We Learned? ............................ 153

\section{Herbig Ae/Be Visual Binaries}

J. Bouvier, P. Corporon

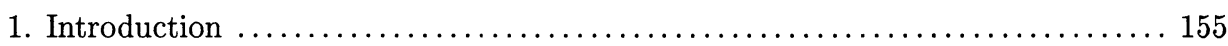

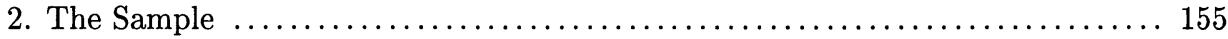

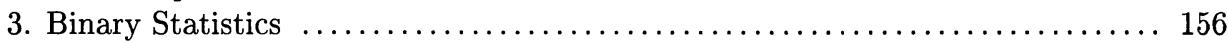

4. Spectrophotometric Properties of the Binary Systems $\ldots \ldots \ldots \ldots \ldots \ldots \ldots \ldots \ldots$

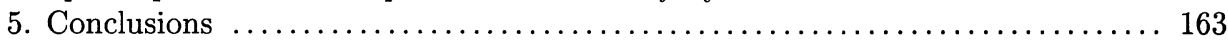
A Spectroscopic and Photometric Survey for Pre-Main Sequence Binaries
E. W. Guenther, V. Joergens, R. Neuhäuser, G. Torres, N. S. Batalha, J. Vijapurkar, M. Fernández, R. Mundt

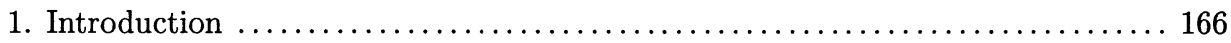

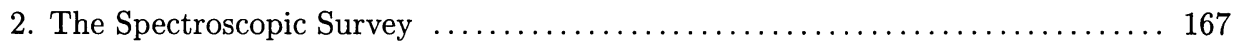

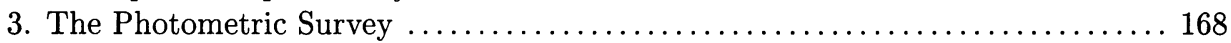




\title{
VI. BINARY POPULATIONS IN YOUNG CLUSTERS
}

\section{Binarity in the Orion Trapezium Cluster}

\author{
M. J. McCaughrean
}

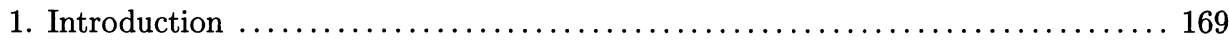

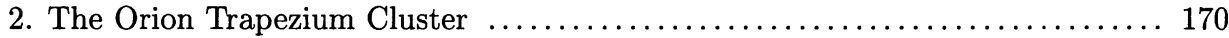

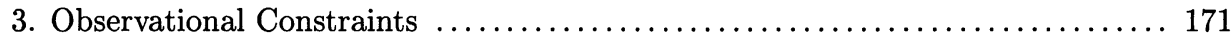

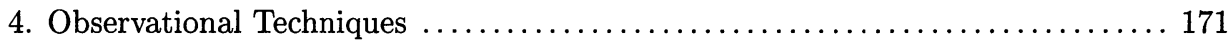

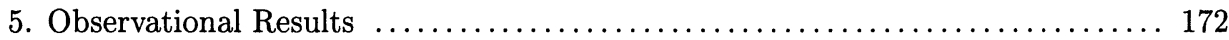

5.1. Close Visual Binaries .................................... 172

5.2. Extending to Wide Binaries ................................ 175

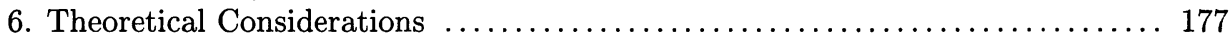

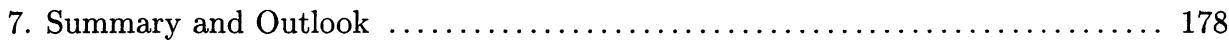

\section{The Properties of Open Cluster Binaries Based on High-Resolution Imaging Surveys}

J. Patience, G. Duchêne

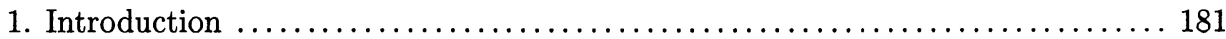

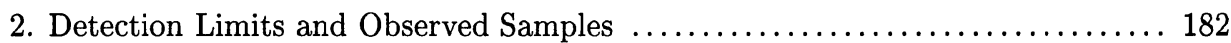

2.1. Observational Techniques .................................. 182

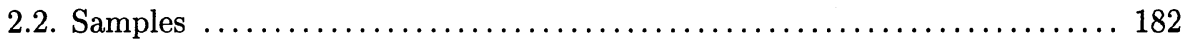

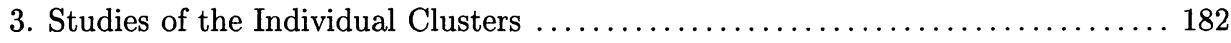

3.1. Binary Fraction as a Function of Age $\ldots \ldots \ldots \ldots \ldots \ldots \ldots \ldots \ldots \ldots \ldots \ldots \ldots$

3.2. Binary Fraction as a Function of Stellar Density .................. 183

3.3. Dynamical Evolution of Young Clusters $\ldots \ldots \ldots \ldots \ldots \ldots \ldots \ldots \ldots \ldots \ldots \ldots$

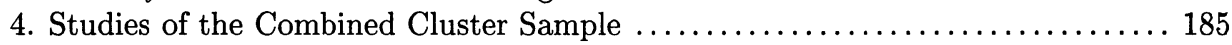

4.1. Observational Properties ................................. 185

4.2. Comparisons with Binary Star Formation Models ................ 187

4.3. Effects of Companions on Stellar Properties $\ldots \ldots \ldots \ldots \ldots \ldots \ldots \ldots \ldots . \ldots 187$

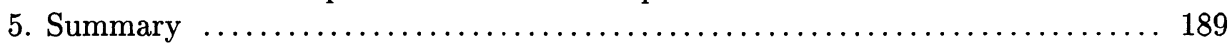

\section{Spectroscopic Binaries in Young Open Clusters}

J.-C. Mermilliod, B. Garcia

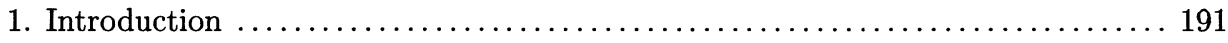

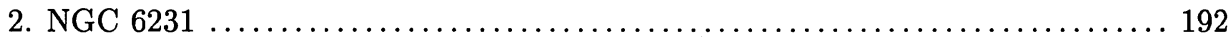

3. Binary Frequency of O-Type Stars in Galactic Open Clusters ............. 192

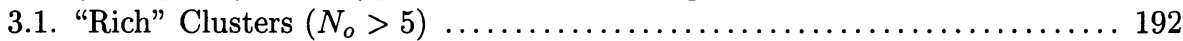

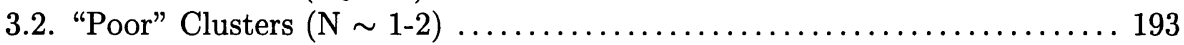

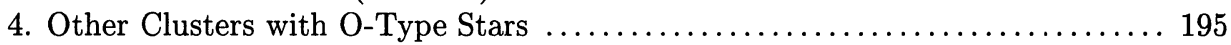

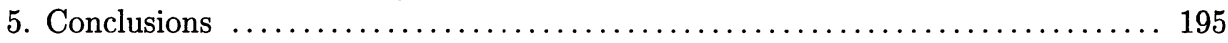

\section{Binary Stars in Young Clusters - a Theoretical Perspective} P. Kroupa

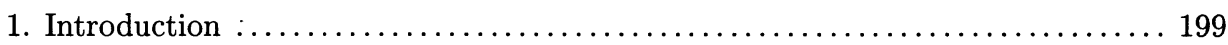

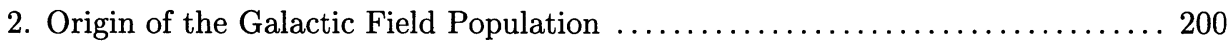


3. The Binary Star - Star Cluster Connection: Fundamentals $\ldots \ldots \ldots \ldots \ldots \ldots . \ldots 201$

4. Forbidden Orbits - a Window to Stellar Evolution . . . . . . . . . . . . . . 206

\section{Summary Talk: What Can Pre-Main Sequence Binary Star} Populations Tell Us about Binary Formation Mechanisms?

A. M. Ghez

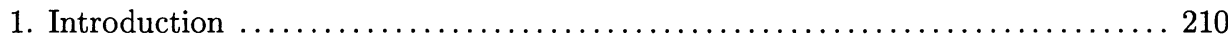

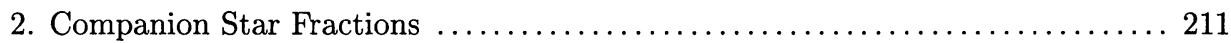

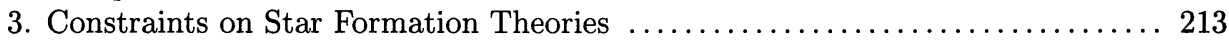

\section{ENVIRONMENTS OF YOUNG BINARIES - DIRECT IMAGING}

\section{Interferometric Observations of Disks around PMS Binary Stars}

A. Dutrey

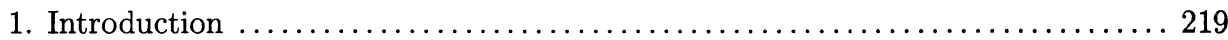

2. Observed Morphology of Material around Multiple T Tauri Systems ....... 220

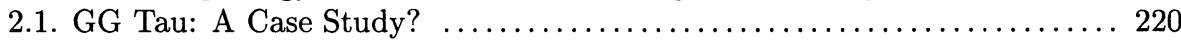

2.2. UY Aur: A Common $0.9^{\prime \prime}$ Binary? . ......................... 221

2.3. UZ Tau: A Quadruple System? ............................ 221

3. Imaging Disks around Binaries with mm Arrays $\ldots \ldots \ldots \ldots \ldots \ldots \ldots \ldots \ldots . \ldots \ldots 22$

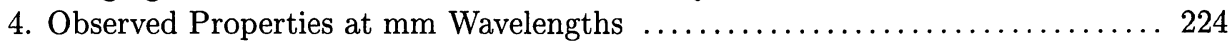

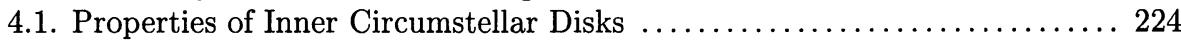

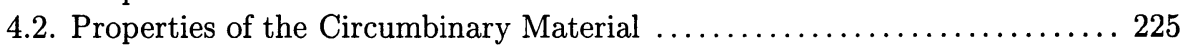

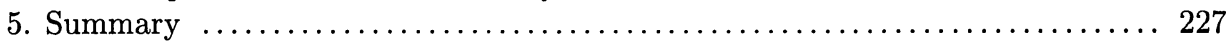

Featured Object: GG Tau - the Ringworld Revisited

S. Guilloteau, A. Dutrey

Optical and Near-Infrared Imaging of Young Binary Star Environments

F. Ménard, K. Stapelfeldt

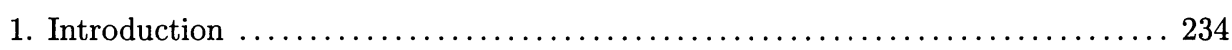

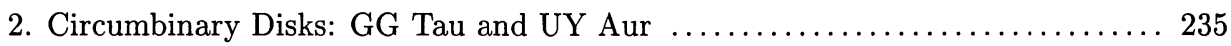

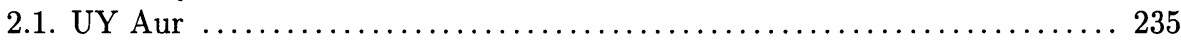

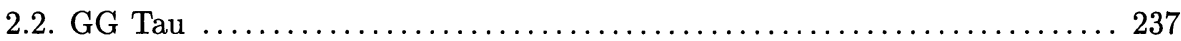

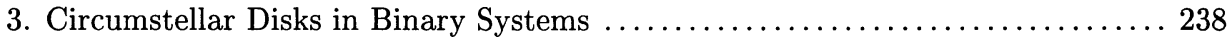

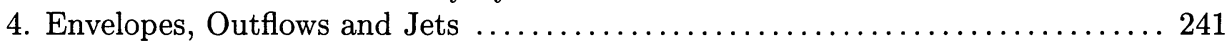

4.1. T Tauri, the Prototype for Young Stellar Object Complexity .......... 241

4.2. Large Envelopes: The Case of CoKu Tau/1 .................... 241

4.3. Mass Loss: RW Aurigae \& XZ Tauri ....................... 242

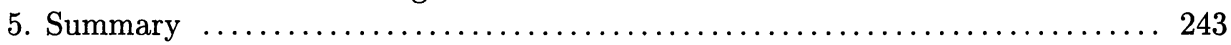


Featured Object:

A Detailed Study of the GG Tau Circumbinary Disk

C.-E. McCabe, A. M. Ghez

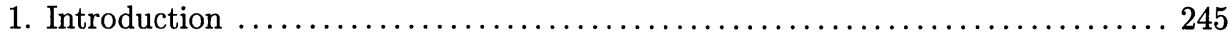

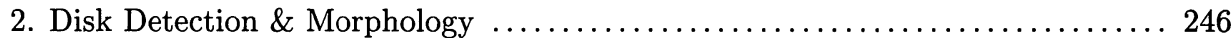

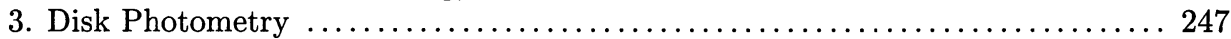

\section{Outflows from Newborn Multiple Stars}

B. Reipurth

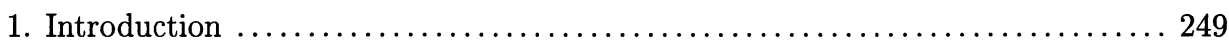

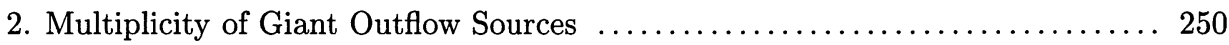

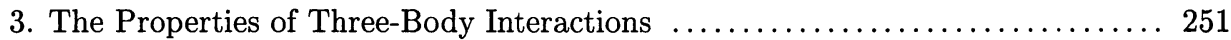

4. Disk-Disk Interactions and Giant HH Bow Shocks $\ldots \ldots \ldots \ldots \ldots \ldots \ldots \ldots \ldots \ldots$

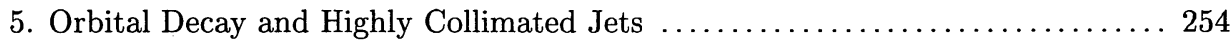

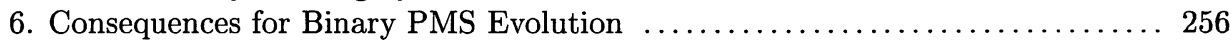

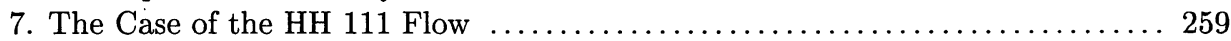

\section{Featured Object: Twisted Jets from L1551-IRS5}

\section{Y. Itoh}

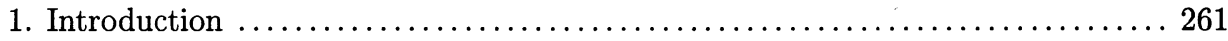

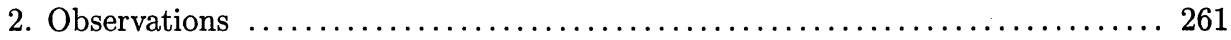

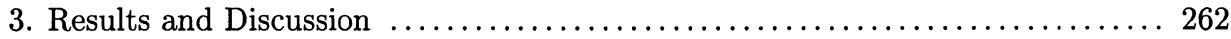

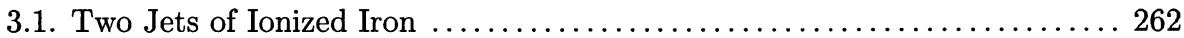

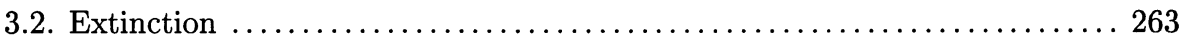

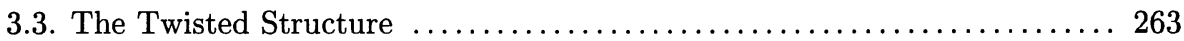

\section{The Infrared Companions of T Tauri Stars:}

Clues to the Formation and Early Evolution of Binaries

C. D. Koresko, C. Leinert

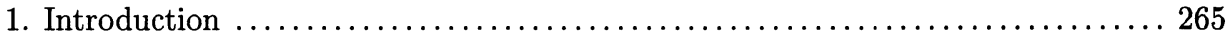

2. Properties of the Infrared Companions ............................ 266

2.1. Infrared Excess and Bolometric Temperature ................... 266

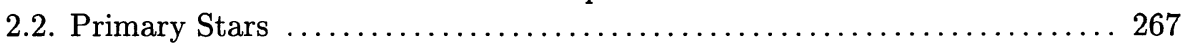

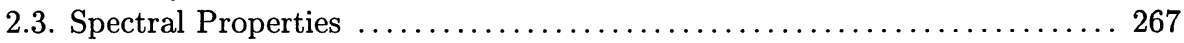

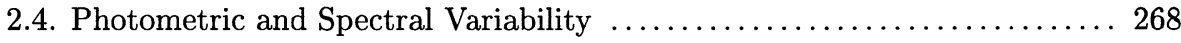

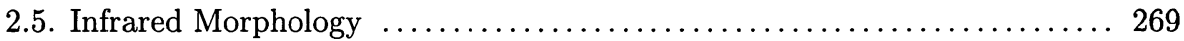

2.6. Radio Emission and Circumstellar Matter ...................... 269

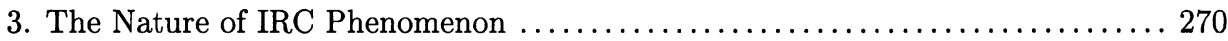

3.1. Are the IRCs Coeval with their Primaries? $\ldots \ldots \ldots \ldots \ldots \ldots \ldots \ldots \ldots . \ldots \ldots$

3.2. Special Viewing Geometries ................................ 271

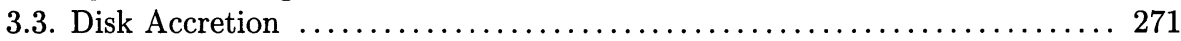

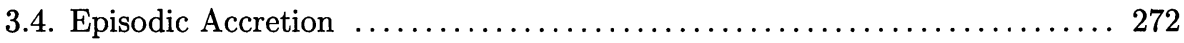

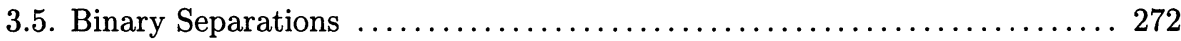

4. Implications for the Formation and Evolution of Binary Stars ............ 272 
Featured Object:

The Close Environment of Z CMa Binary System

P. J. V. Garcia

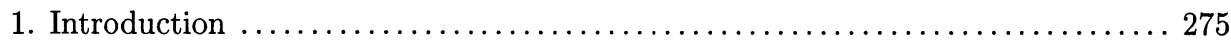

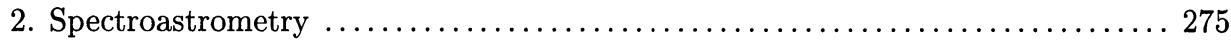

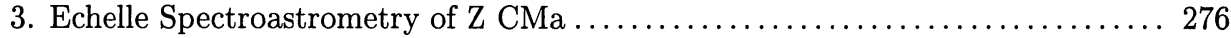

Statistics of Binary and Multiple Young Stars Observed by Lunar Occultations

A. Richichi

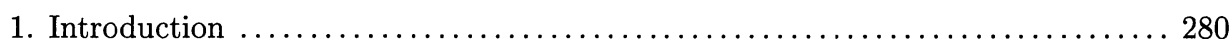

2. Lunar Occultations for Studies of Young Binary Stars $\ldots \ldots \ldots \ldots \ldots \ldots \ldots 280$

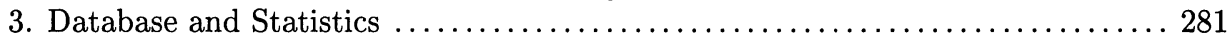

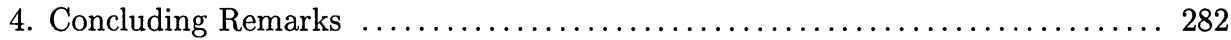

\section{ENVIRONMENTS OF YOUNG BINARIES - INDIRECT OBSERVATIONS}

\section{Disks in Young Binary Systems: \\ Unresolved Millimeter-Wave Observations}

E. L. N. Jensen

1. Introduction .............................................. 285

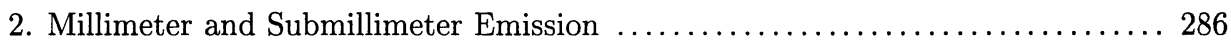

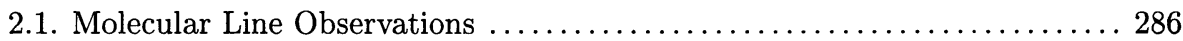

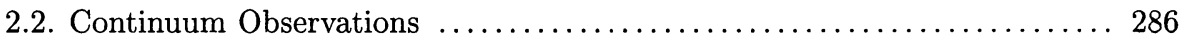

3. Millimeter Flux as a Function of Binary Separation $\ldots \ldots \ldots \ldots \ldots \ldots \ldots \ldots 286$

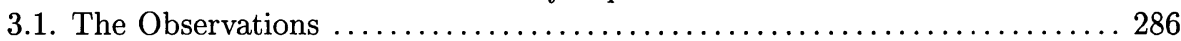

3.2. Disk Truncation ..................................... 288

3.3. Infrared Fluxes and the Presence of Circumstellar Disks ............. 290

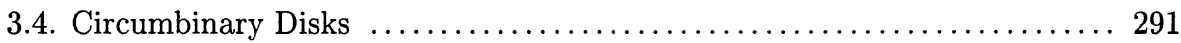

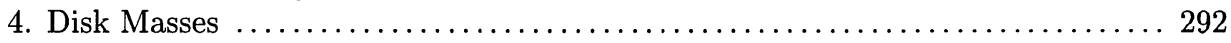

5. Implications for Planet Formation in Binary Systms . ................ 292

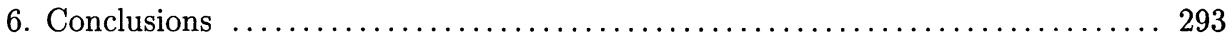

\section{Pre-Main Sequence Binaries with Aligned Disks?}

S. Wolf, B. Stecklum, T. Henning

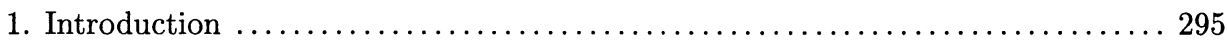

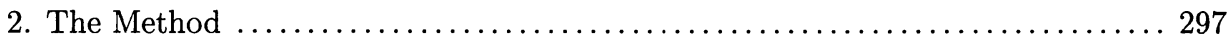

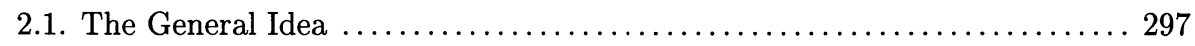

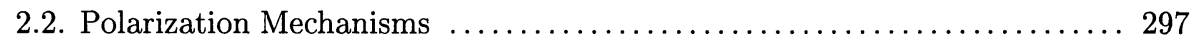

2.3. Inclination of Disks - Projection Effect . . . . . . . . . . . . . . . . . . 299

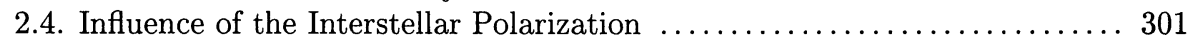




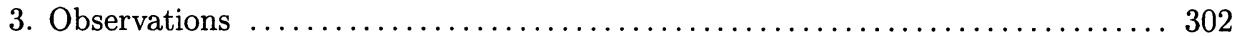

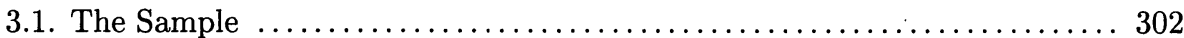

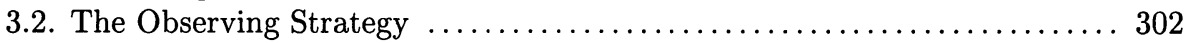

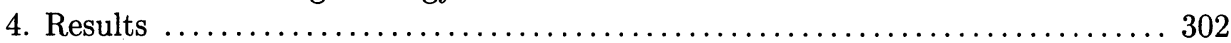

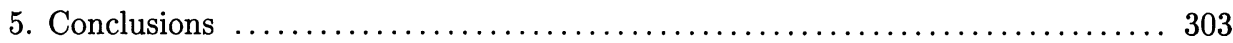

Doppler Imaging as a Test for Binary Star Formation Theories

K. G. Strassmeier

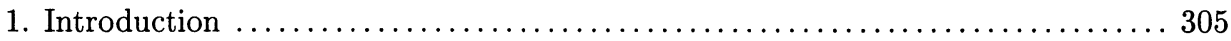

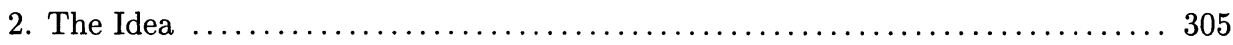

3. Testing the Inclination Sensitivity of Doppler Imaging $\ldots \ldots \ldots \ldots \ldots \ldots \ldots \ldots$

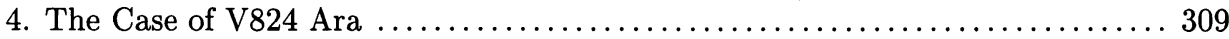

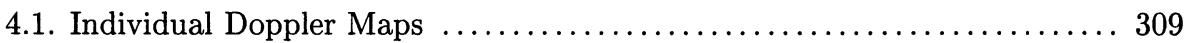

4.2. The Spin Inclinations of the Binary Components $\ldots \ldots \ldots \ldots \ldots \ldots \ldots 311$

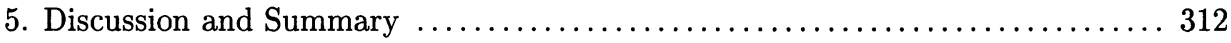

Correlations of Spectral Accretion Signatures in Young Binaries L. Prato, J.-L. Monin

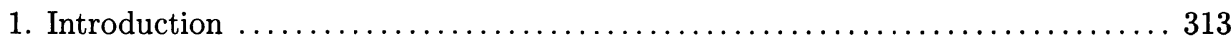

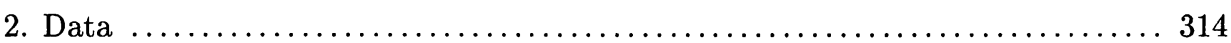

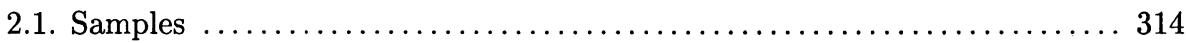

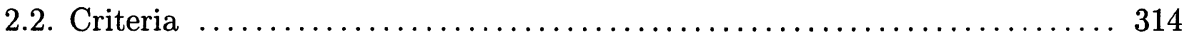

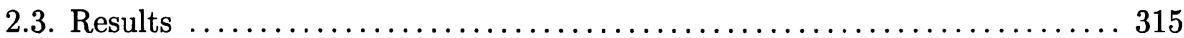

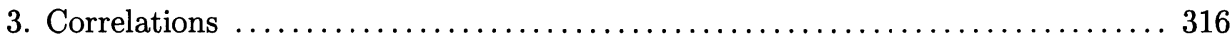

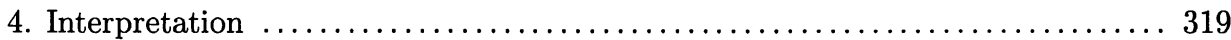

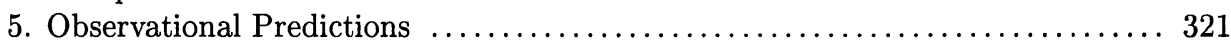

\section{First Results from a STIS Survey} of Close Pre-Main Sequence Binaries in Taurus

S. J. Kenyon, P. Hartigan

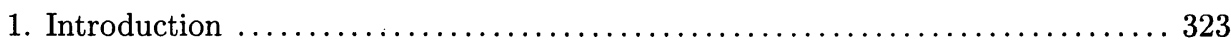

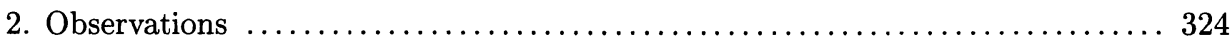

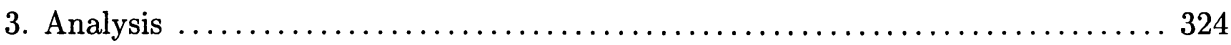

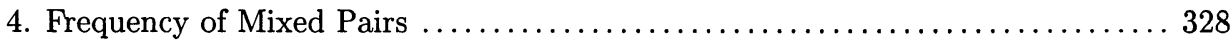

5. Ages of Binary Components ................................... 329

\section{Young Binary Stars in Taurus-Auriga}

R. J. White, A. M. Ghez

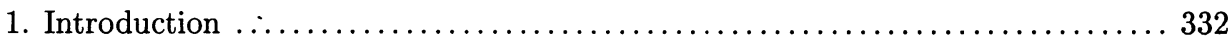

2. High Spatial Resolution Imaging and Spectroscopy $\ldots \ldots \ldots \ldots \ldots \ldots \ldots \ldots \ldots \ldots$

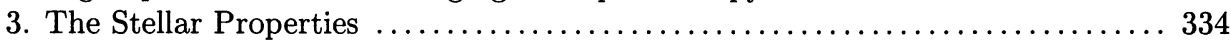

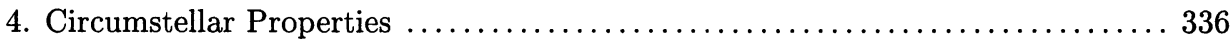

4.1. Circumprimary and Circumsecondary Disks $\ldots \ldots \ldots \ldots \ldots \ldots \ldots \ldots \ldots \ldots$

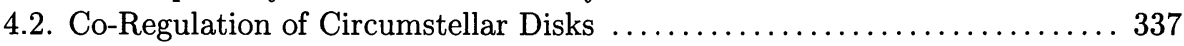

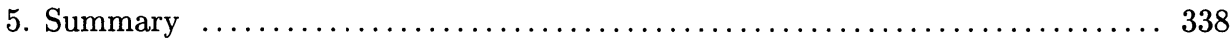


Featured Object: Disk Clearing in the Young Binary AK Sco E. L. N. Jensen

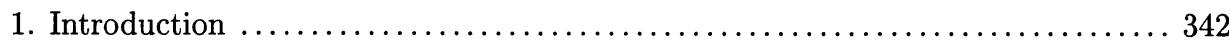

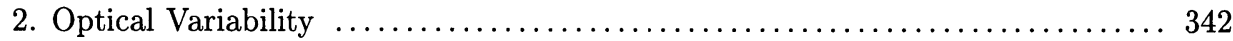

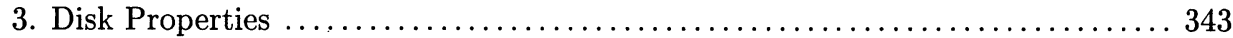

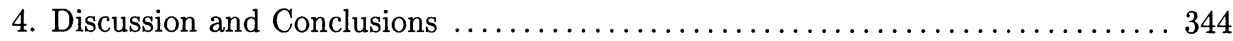

Summary Talk: What Can Observations of Young Binaries Tell Us about Binary Formation?

C. J. Clarke

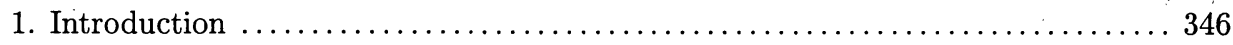

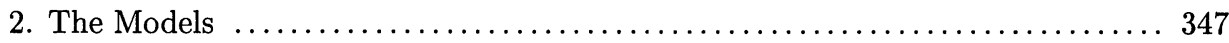

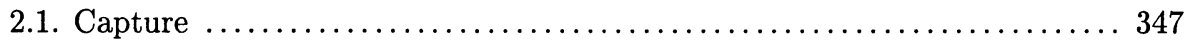

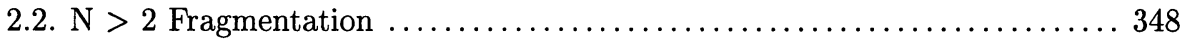

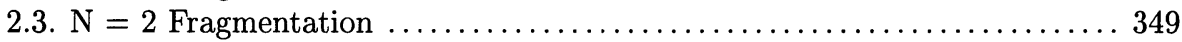

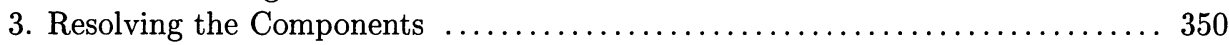

4. Synthesizing the Field - Are the Wide Binaries a Problem? ............. 352

Photographic Intermezzo $\ldots \ldots \ldots \ldots \ldots \ldots \ldots \ldots \ldots \ldots \ldots \ldots \ldots \ldots \ldots$

\section{THEORETICAL CONTEXT - DETAILED CALCULATIONS}

\section{Fragmentation and Star Formation in Turbulent Cores}

R. I. Klein, R. Fisher, C. F. McKee

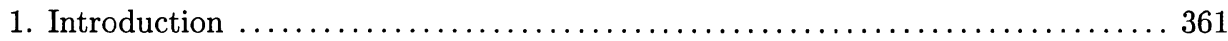

2. Basic Questions to Be Addressed .............................. 362

3. The Key Role of Nonlinear Perturbations: A Numerical Experiment ........ 363

4. Turbulent Low-Mass Molecular Cloud Cores: Initial Conditions ............ 365

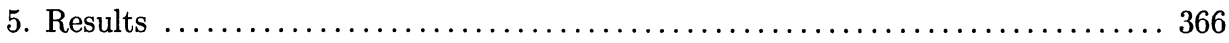

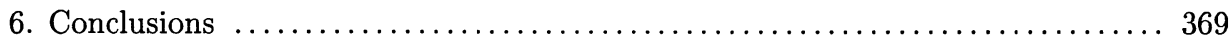

\section{Impact of Magnetic Fields on Fragmentation}
A. P. Boss

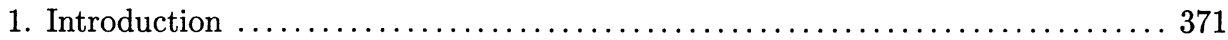

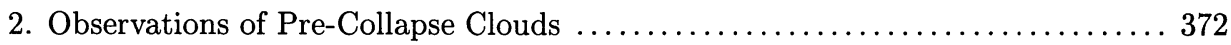

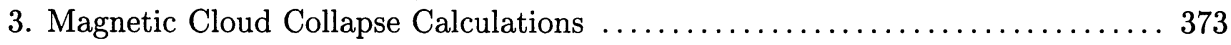

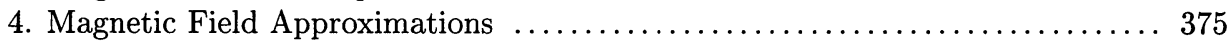

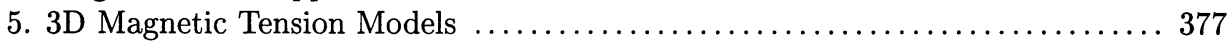

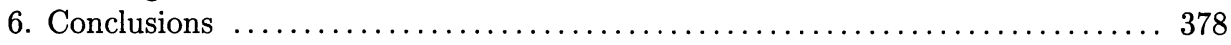




\section{Can Disks Produce Companions by Gravitational Fragmentation?}

\section{R. H. Durisen}

1. Why Ask the Question? ....................................... 381

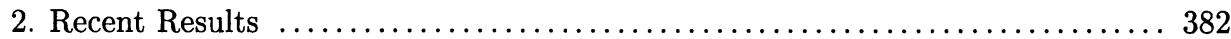

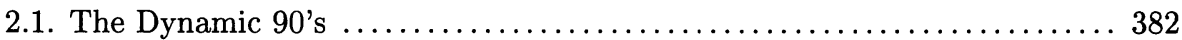

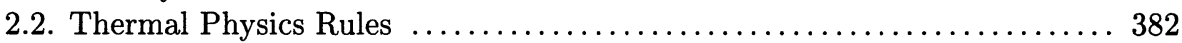

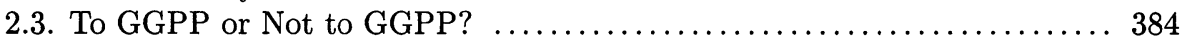

2.4. Heating, Cooling, and the Third Spatial Dimension $\ldots \ldots \ldots \ldots \ldots \ldots . \ldots 386$

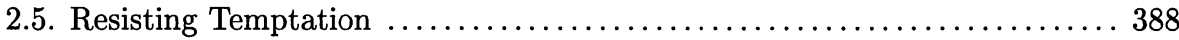

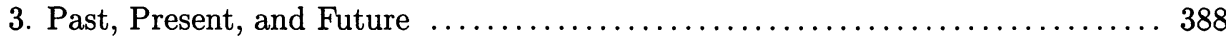

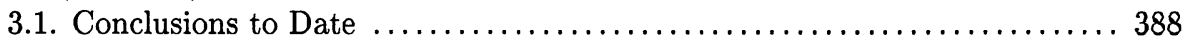

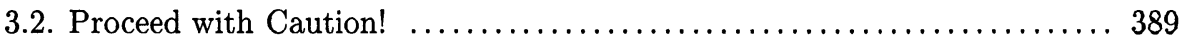

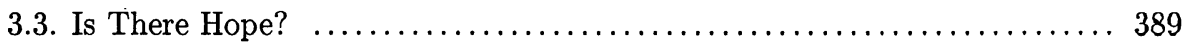

\section{Filament Fragmentation}

S. Inutsuka, T. Tsuribe

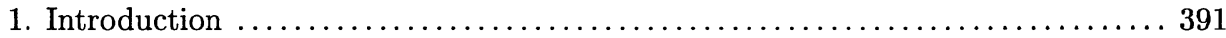

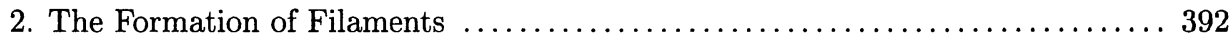

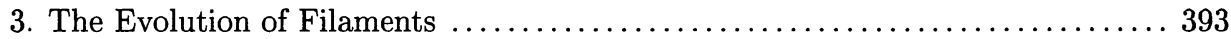

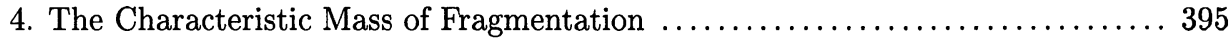

5. Mass Function of Molecular Cloud Cores . . . . . . . . . . . . . . . . . . . . . 397

6. Mean Surface Density of Companions .......................... 398

\section{Outflow from Protostars and Angular Momentum Transfer}
K. Tomisaka
1. Introduction 401

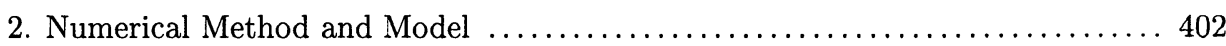
3. Run-Away Collapse Stage $\ldots \ldots \ldots \ldots \ldots \ldots \ldots \ldots \ldots \ldots \ldots \ldots \ldots \ldots \ldots \ldots . \ldots \ldots 2$

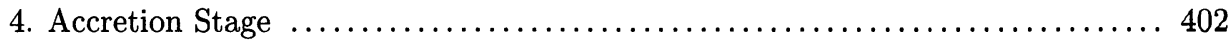
5. Angular Momentum Transfer .................................. 404

\section{Tidally-Induced Angular Momentum Transport in Disks}

C. E. J. M. L. J. Terquem

1. Angular Momentum Exchange between the Disk Rotation and the Orbital

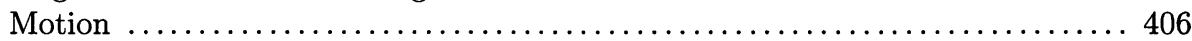

2. Angular Momentum Transport by Density Waves .................... 407

3. Angular Momentum Transport by Bending Waves .................. 408

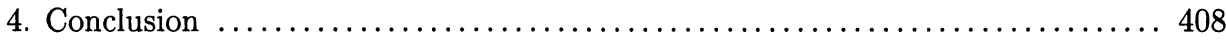




\section{Angular Momentum Transport and Dynamo Effect in Kepler Disks}

G. Rüdiger, U. Ziegler

1. Angular Momentum Transport ................................ 410

2. Shear Flow Dynamo of a Differentially Rotating Sphere ................ 411

3. Local Simulations of a Differentially Rotating Keplerian Disk.............. 412

\section{EVOLUTION OF YOUNG BINARY SYSTEMS}

\section{Featured Object: A Brief Introduction to DQ Tau}

K. G. Stassun

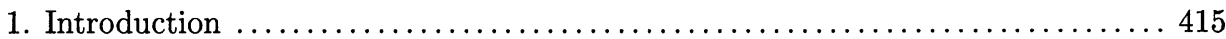

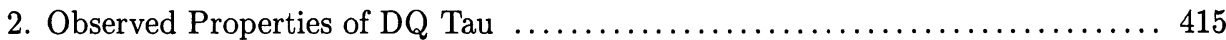

2.1. A "Classical" Classical T Tauri Star? ........................ 415

2.2. A CTTS SB2 - not so Typical after all! ...................... 415

3. A Clue: Periodic "Accretion Events" in DQ Tau ..................... 416

3.1. Periodic Photometric Brightenings .......................... 416

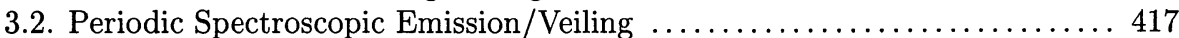

4. DQ Tau: Exemplar for Orbitally Driven Accretion in Close Binaries ........ 417

\section{Accretion onto Pre-Main Sequence Binary Stars: The Circumstellar Environment}

R. D. Mathieu

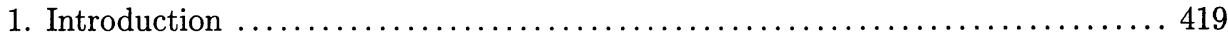

2. Case Studies: Challenges for Disk-Clearing Theory $\ldots \ldots \ldots \ldots \ldots \ldots \ldots \ldots \ldots 421$

2.1. Challenge 1: UZ Tau E ................................... 421

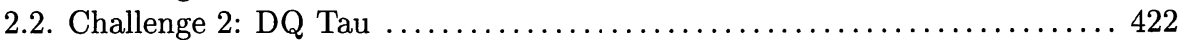

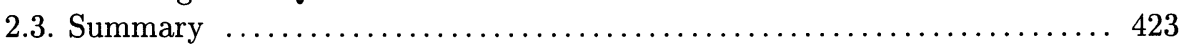

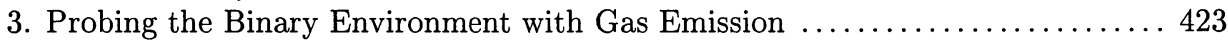

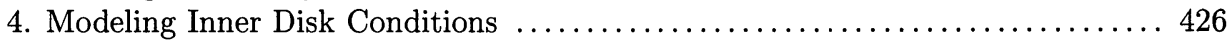

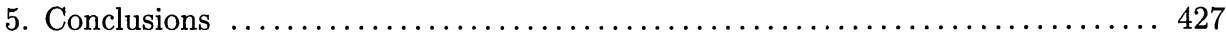

\section{Accretion and the Properties of Protobinary Systems}

M. R. Bate

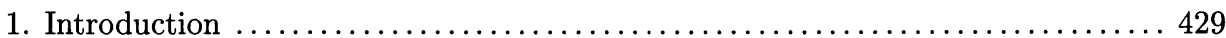

2. The Evolution of Accreting Protobinary Systems $\ldots \ldots \ldots \ldots \ldots \ldots \ldots \ldots \ldots$. 430

2.1. The Quantitative Effects of Accretion ...................... 430

2.2. A Protobinary Evolution Code (PBE Code) $\ldots \ldots \ldots \ldots \ldots \ldots \ldots \ldots \ldots \ldots$

3. Observational Implications: Isolated Star Formation $\ldots \ldots \ldots \ldots \ldots \ldots \ldots \ldots \ldots 44$

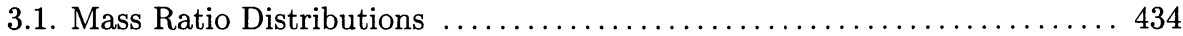

3.2. Circumstellar Discs ...................................... 435

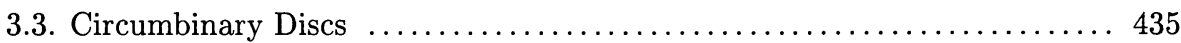


xviii

4. Observational Implications: Clustered Star Formation $\ldots \ldots \ldots \ldots \ldots \ldots \ldots \ldots 436$

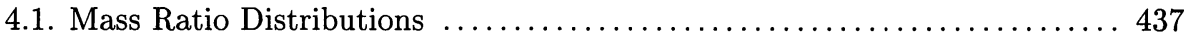

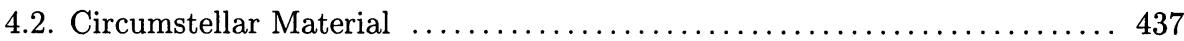

5. Conclusions ................................................ 437

Early Orbital Evolution of Binaries: Overview and Outlook P. Artymowicz, S. H. Lubow

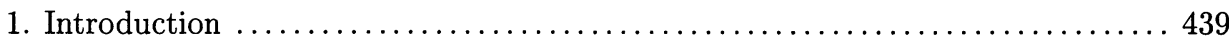

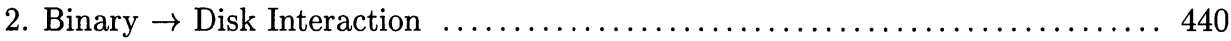

2.1. Eccentric Binaries and the Mass Transfer ..................... 440

2.2. Time-Dependent Streaming through the Gap .................... 440

2.3. Non-Coplanar and 3-D Disks ............................. 442

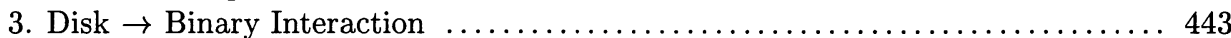

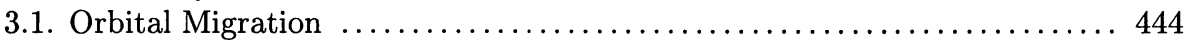

3.2. Eccentricity: Damped or Pumped up? ...................... 444

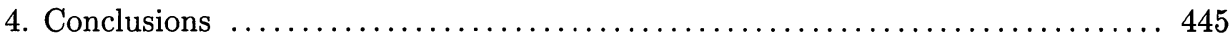

\section{YOUNG BINARIES AND STELLAR ASTROPHYSICS}

\section{Featured Object:}

The Intriguing PMS Eclipsing (Triple) System TY CrA

L. P. R. Vaz

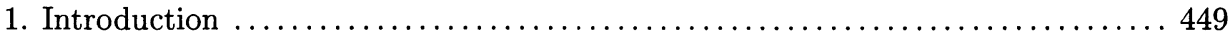

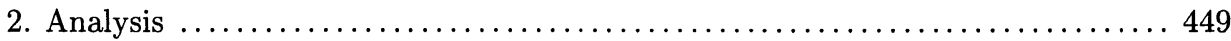

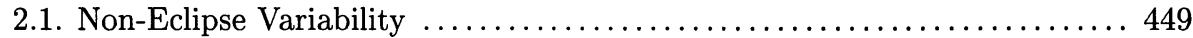

2.2. Rotational Velocity and Tertiary Component $\ldots \ldots \ldots \ldots \ldots \ldots \ldots \ldots \ldots 450$

3. Evolutionary Status .......................................... 451

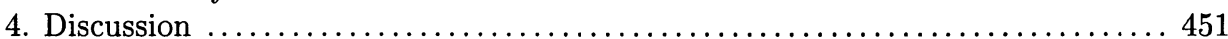

\section{Measuring the Masses of Young Stars}

\section{Simon}

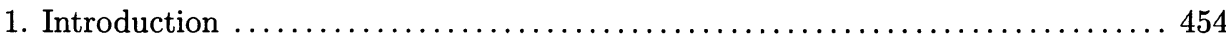

2. Dynamical Methods of Mass Measurement ....................... 455

3. Application to Young Visual Binaries and Spectroscopic Binaries ......... 456

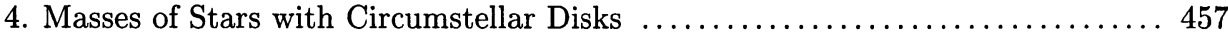

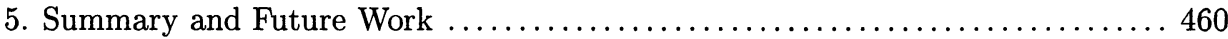

Featured Object:

A Relative Age Test of Pre-Main Sequence Evolutionary Models Based on the Young Quadruple System GG Tauri

R. J. White

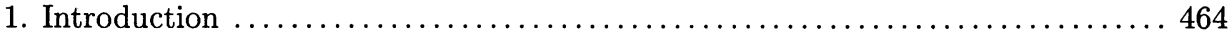

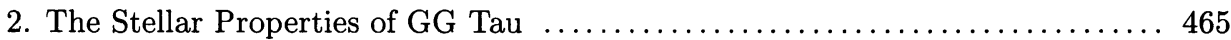

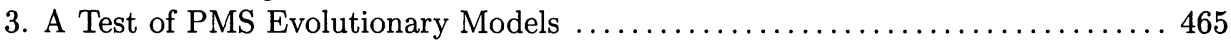


Featured Object: The PMS Eclipsing Binary RXJ 0529.4+0041

E. Covino, S. Catalano, A. Frasca, E. Marilli, J. M. Alcalá, M. Fernández, C. Melo, R. Paladino, B. Stelzer

1. Introduction .............................................. 468

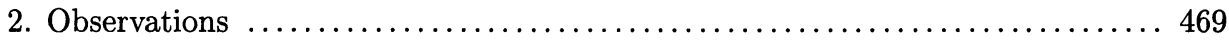

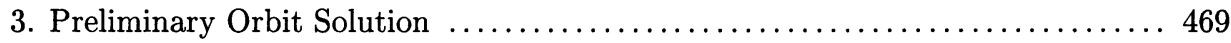

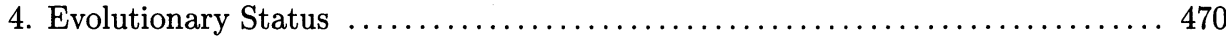

Testing Pre-Main Sequence Models with Young Binaries

F. Palla

1. Tests of PMS Evolutionary Tracks $\ldots \ldots \ldots \ldots \ldots \ldots \ldots \ldots \ldots \ldots \ldots \ldots \ldots, \ldots \ldots \ldots$

2. Estimating Stellar Masses: Single Stars $\ldots \ldots \ldots \ldots \ldots \ldots \ldots \ldots \ldots \ldots \ldots \ldots 474$

3. Estimating Stellar Masses: PMS Binary Stars $\ldots \ldots \ldots \ldots \ldots \ldots \ldots \ldots \ldots \ldots 475$

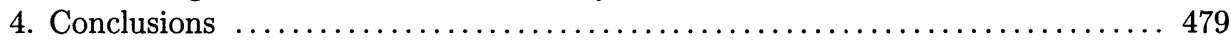

\section{INVITED DEBATE: THE STATUS OF PMS TRACKS}

\section{Pre-Main Sequence Models} for Low-Mass Stars and Brown Dwarfs

I. Baraffe, G. Chabrier, F. Allard, P. Hauschildt

1. Introduction ............................................. 483

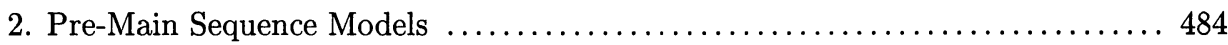

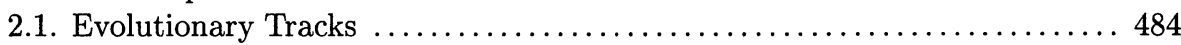

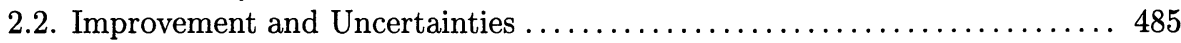

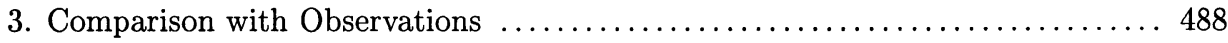

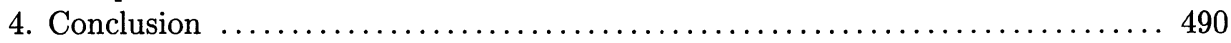

\section{A Dialogue on Dynamical Pre-Main Sequence Tracks \\ G. Wuchterl}

A Fictional but Scientific Debate

\section{Unresolved Pre-Main Sequence Binaries in the HR Diagram}

\section{P. Hartigan, S. Kenyon}

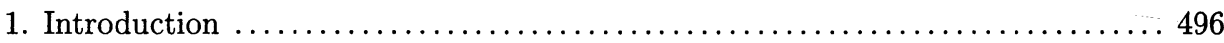

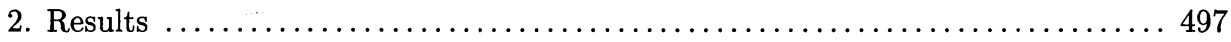

2.1. Case 1: Equal Components ............................. 497

2.2. Case 2: Different Components, Faint Secondary ................... 497

2.3. Case 3: Different Components, Bright Secondary .................. 499

2.4. Case 4: Different Components, Differing Reddening Procedures ........ 499

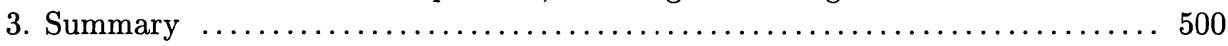




\section{The Effect of Unresolved Binaries on the Low-Mass IMF}

O. Malkov, H. Zinnecker

1. Monte Carlo Simulations ...................................... 501

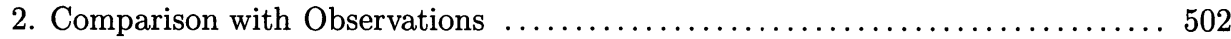

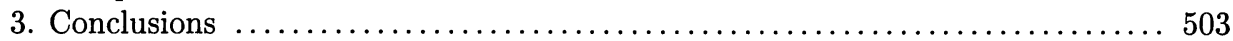

\section{Fun for Two}

S. F. Portegies Zwart, L. R. Yungelson, G. Nelemans

1. Introduction .............................................. 505

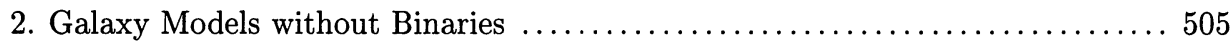

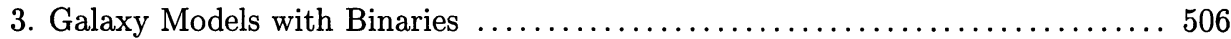

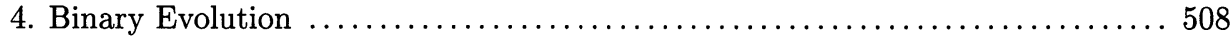

5. Why Are the Models so Different? .............................. 509

\section{PLANET FORMATION IN BINARY SYSTEMS}

\section{Planet Formation in Binary Systems}

W. Kley

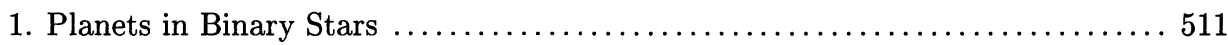

2. Influence of Companions on Disks ............................ 512

3. On the Formation of Planets in Tidally Perturbed Disks $\ldots \ldots \ldots \ldots \ldots \ldots \ldots 512$

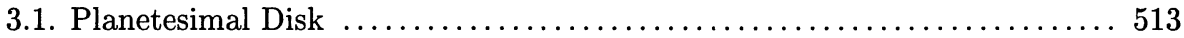

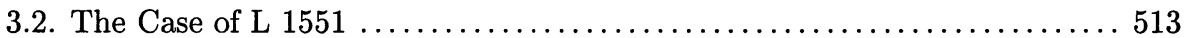

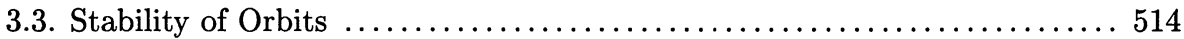

3.4. The Evolution of an Embedded Planet in a Binary Star .............. 516

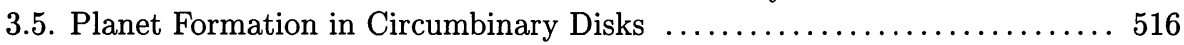

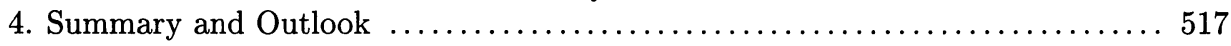

\section{Comparison between Extrasolar Planets and} Low-Mass Secondaries

T. Mazeh, S. Zucker

1. Introduction ............................................... 519

2. Eccentricity and Period Distribution ........................... 521

3. The Mass Distribution of the Planet Candidates and the Low-Mass Secondaries 523

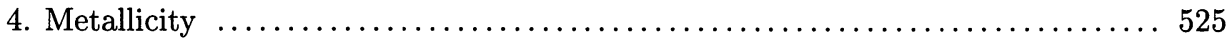

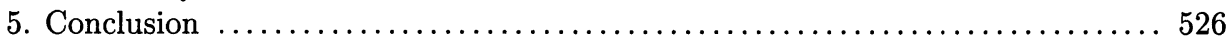




\section{Microlensing: A Tool to Probe Distant Binary Populations}

R. Di Stefano

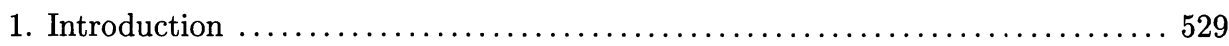

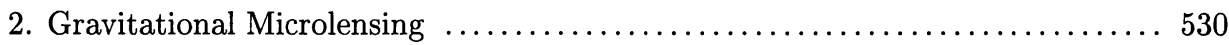

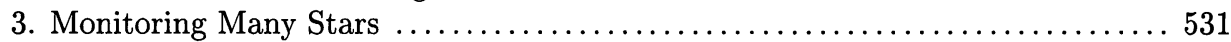

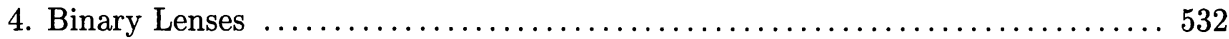

4.1. Most of the Lenses May Be Binaries ........................ 533

4.2. The Magellanic-Cloud Events .............................. 533

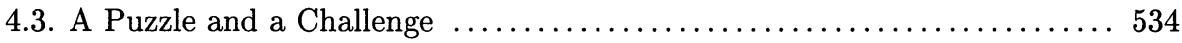

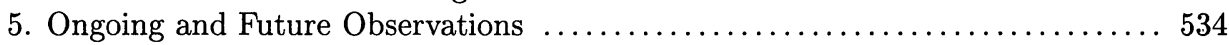

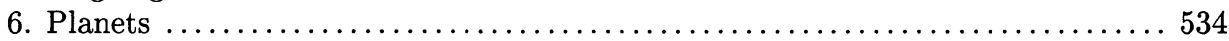

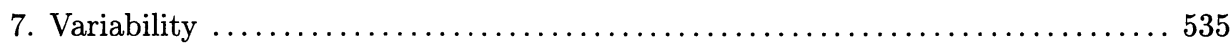

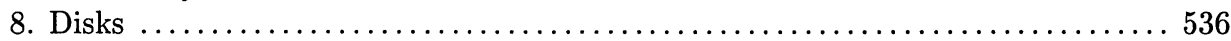

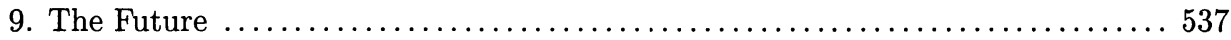

\section{Optical and Infrared Long-Baseline Interferometry:} Application to Binary Star Science

\section{A. Quirrenbach}

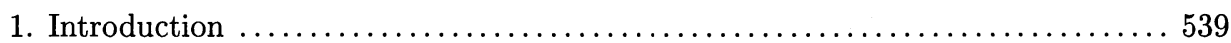

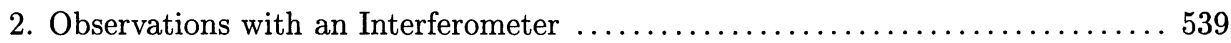

3. Combination of Interferometry with other Methods $\ldots \ldots \ldots \ldots \ldots \ldots \ldots \ldots \ldots$

4. Stellar Masses and Tests of Evolutionary Models .................... 542

5. Low-Mass and Sub-Stellar Companions .......................... 543

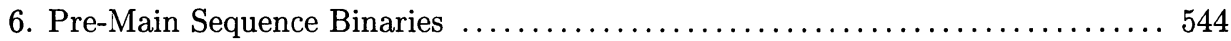

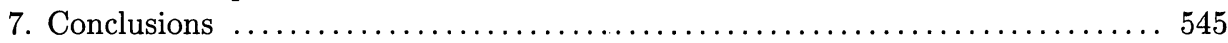

\section{The Future of $\mathrm{mm} /$ submm Interferometry: The ALMA Project}

S. Guilloteau

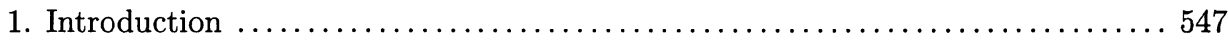

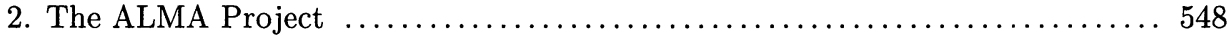

3. ALMA and Multiple Stellar System Formation $\ldots \ldots \ldots \ldots \ldots \ldots \ldots \ldots \ldots \ldots \ldots$

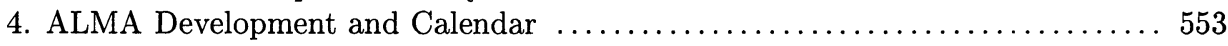

\section{Adaptive Optics Imaging of Faint Companions:}

\section{Current \& Future Prospects}

L. M. Close

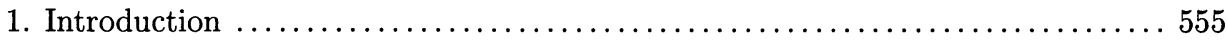

2. Observational Procedure to Detect Faint Companions with AO \& an Example Result with MWC480 .................................... 556

3. The Future: High Spatial \& Low Spectral Resolution with AO . .......... 558

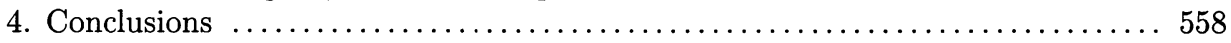


xxii

Future Opportunities in Young Binary Star Research with Space Observatories

K. Stapelfeldt

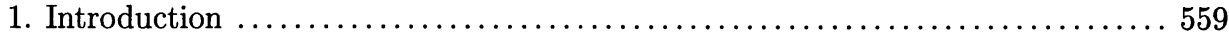

2. Current Facilities ......................................... 559

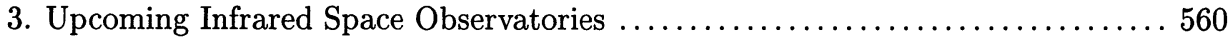

4. Upcoming Space Astrometry Missions ............................... 562

Numerical Hydrodynamics: SPH versus AMR

T. Plewa

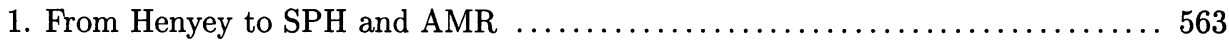

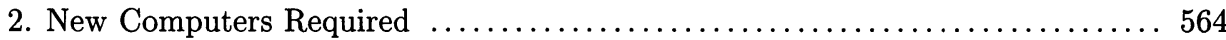

3. A Few Critical Comments ...................................... 565

Frontiers of Radiative Transfer

T. Henning

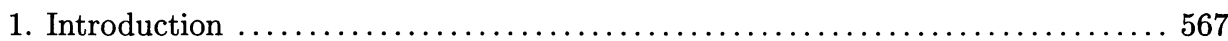

2. Radiative Transfer - More Dimensions . . . . . . . . . . . . . . . . . . . . . 568

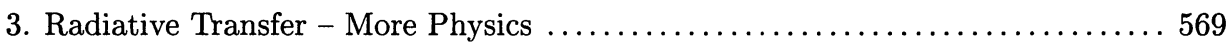

4. Conclusions and Outlook .................................... 570

XV. HIGHLIGHTS AND FAREWELL

Impressions of IAU 200

R. Ebert

More Impressions (Photos) f........................... 575

Summary and Reflections

R. D. Mathieu

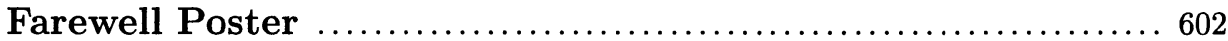

